Engineering with Computers manuscript No.

(will be inserted by the editor)

\section{Simultaneous aligning and smoothing of surface triangulations}

\author{
José M. Escobar . \\ Rafael Montenegro . \\ Eduardo Rodríguez . \\ Gustavo Montero
}

Received: 27 Febrary 2009 / Accepted: date

\begin{abstract}
In this work we develop a procedure to deform a given surface triangulation to obtain its alignment with interior curves. These curves are defined by splines in a parametric space and, subsequently, mapped to the surface triangulation. We have restricted our study to orthogonal mapping, so we require the curves to be included in a patch of the surface that can be orthogonally projected onto a plane (our parametric space). For example, the curves can represent interfaces between different materials or boundary conditions, internal boundaries or feature lines. Another setting in which this procedure can be used is the adaption of a reference mesh to changing curves in the course of an evolutionary process. Specifically, we propose a new method that moves the nodes of the mesh, maintaining its topology, in order to achieve two objectives simultaneously: the piecewise approximation of the curves by edges of the surface triangulation and the optimization of the resulting mesh. We will designate this procedure as projecting/smoothing method and it is based on the smoothing technique that we have introduced for surface triangulations in previous works. The mesh quality improvement is obtained by an iterative process where each free node is moved to a new position that minimizes a certain objective function. The minimization process is done on the parametric plane attending to the surface piece-wise approximation and to an algebraic quality measure (mean ratio) of the set of triangles that are connected to the free node. So, the 3-D local projecting/smoothing problem is re-
\end{abstract}

J.M. Escobar · R. Montenegro · E. Rodríguez $\cdot$ G. Montero Institute for Intelligent Systems and Numerical Applications in Engineering, University of Las Palmas de Gran Canaria, Campus Universitario de Tafira, 35017 Las Palmas de Gran Canaria, Spain

Tel.: +34-928457400

Fax: +34-928457401

E-mail: \{jmescobar,rmontenegro,erodriguez,gmontero\}@ siani.es duced to a 2-D optimization problem. Several applications of this method are presented.

Keywords Mesh alignment · Mesh adaptation - Surface mesh smoothing $\cdot$ Node movement $\cdot$ R-adaptivity

\section{Introduction}

The numerical simulation of physical problems requires the internal boundaries and discontinuities to be properly represented. Usually, the largest errors are introduced in a neighborhood of such discontinuities. These errors are often greatly reduced if the mesh is aligned with the discontinuities. That is why it is desirable to have a procedure capable of deforming a given triangulation to get its alignment with a curve. Although there are numerous works dealing with surface mesh optimization, see for example [10,11], only a few of them address the problem of the exact mesh alignment with interior curves. In fact, the only paper that we have found in the bibliography tackling this question in similar terms, but for quadrilateral grids is [13]. The authors consider the problem of aligning a planar grid with multiple embedded curves defined by basic segments as straight lines or arcs of circle. A different approach to the problem can be found in [3], where the curve is approximated by a polygonal line included in the surface triangulation, but in this case the segments are not edges of the mesh. The paper [20] presents a variant of Ruppert's algorithm for producing a 2D Delaunay triangulation of a domain containing arbitrary curved inputs. Nevertheless, this algorithm does not allow a dynamical adaption of the mesh without remeshing.

The work that we describe in this paper is an enhanced version of [7]. The procedure aligns a given surface triangulation with an arbitrary curve and it is based on the surface mesh smoothing technique proposed in [5]. An analytical representation of the curve is not usually available. Instead, it is approximately known by a sequence of interpolating data points. We have chosen a parametric cubic spline as interpolating curve due it is $C^{2}$ continuous and it has other interesting properties that will be used later. Obviously, the grade of approximation of the curve depends on the element sizes, therefore, a good strategy is to combine the projecting/smoothing technique with a local mesh refinement [12]. Our procedure is specially indicated for evolutionary problems where the boundaries change their shape or position with time; for example, the ones related to fluid-structure interactions involving large displacement (see, for example [22]), or crack modeling. The projecting/smoothing technique could be also applied to domain decomposition, definition of material interfaces, free boundary problems, etc.

The organization of the paper is as follows. In section 2 a rough description of the proposed method is presented. In section 3 we propose an objective function, and the cor- 
responding modification, able to untangle and smooth plane triangulations simultaneously. The projecting/smoothing method is initially analyzed for plane triangulations in section 4 and, afterward, it is extended to triangulations defined on curved surfaces in section 5. Section 6 is devoted to applications. The paper concludes with a brief discussion of the work and its possible extensions.

\section{Statement of the Projecting/Smoothing Method}

Let $C$ be a curve, and suppose that it is embedded in a surface triangulation $T_{\Sigma}$ (see Figure 3 ). The basic idea of the projecting/smoothing method lies in relocating the nodes of $T_{\Sigma}$ closest to $C$ in positions just sited in the curve. This operation, which we will refer to as node projection onto the curve, goes on until getting an approximate representation (interpolation) of $C$ by linked edges of $T_{\Sigma}$. A node of $T_{\Sigma}$ is considered projectable if we can displace it from its initial position to any point of $C$ in such a way the local mesh does not get tangled. This projection implies an enforced alteration of the original positions of the nodes and, in general, has a negative effect on the quality of the triangles close to $C$. To avoid this drawback, the remaining nodes are also displaced to new positions following the smoothing procedure proposed in [5].

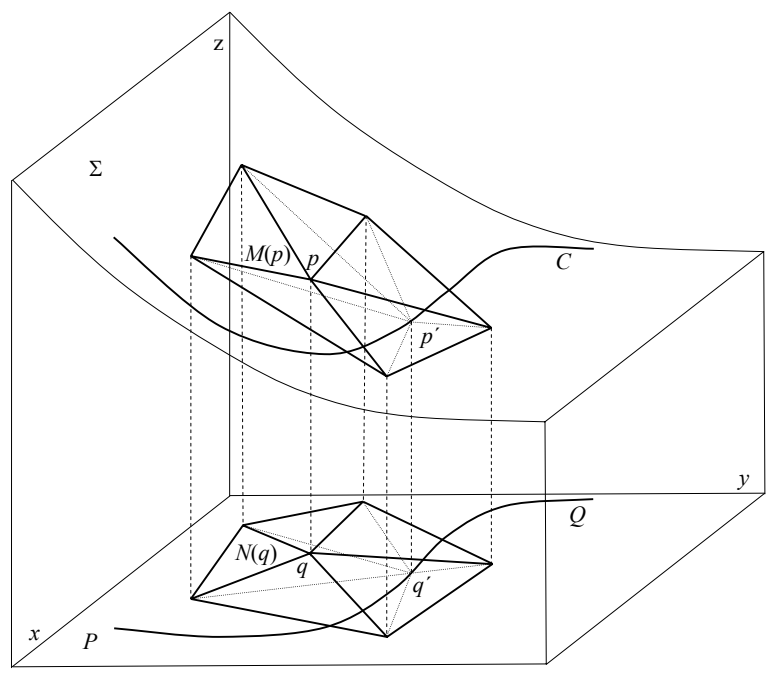

Fig. 1 The relocation of node $p \in \Sigma$ is performed in the plane $P$ by projecting $q$ on $Q$ and, consequently, $p$ on $C$

For 2-D (or 3-D) meshes, the quality improvement can be obtained by an iterative process where each node of the mesh is moved to a new position that minimizes an objective function derived from certain algebraic quality measure of the local mesh [16,9]. The objective function presents a barrier in the boundary of the feasible region associated to the free node. In this context the 2-D (or 3-D) feasible region is the set of points where the free node could be placed to get a valid local mesh, that is, without inverted elements. The barrier has an important role because it avoids the optimization algorithm to create a tangled mesh when it starts with a valid one. We show in [5] a procedure for smoothing surface triangulations taking into account these aspects. The basic idea lies in transforming the original problem on $\Sigma$ into a two-dimensional one on a plane $P$. To do this, the local mesh $M(p)$, belonging to $T_{\Sigma}$, is orthogonally projected onto a plane $P$ performing a local mesh $N(q)$, where $p$ is the free node on $\Sigma$ and $q$ is its orthogonal projection onto $P$. The plane $P$ is suitably chosen in terms of $M(p)$ in order to get a valid mesh on $P$ (see Figure 3 ). Thus, the optimization of $M(p)$ is got by the appropriate optimization of $N(q)$. It involves the construction of ideal triangles in $N(q)$ that become near equilateral in $M(p)$.

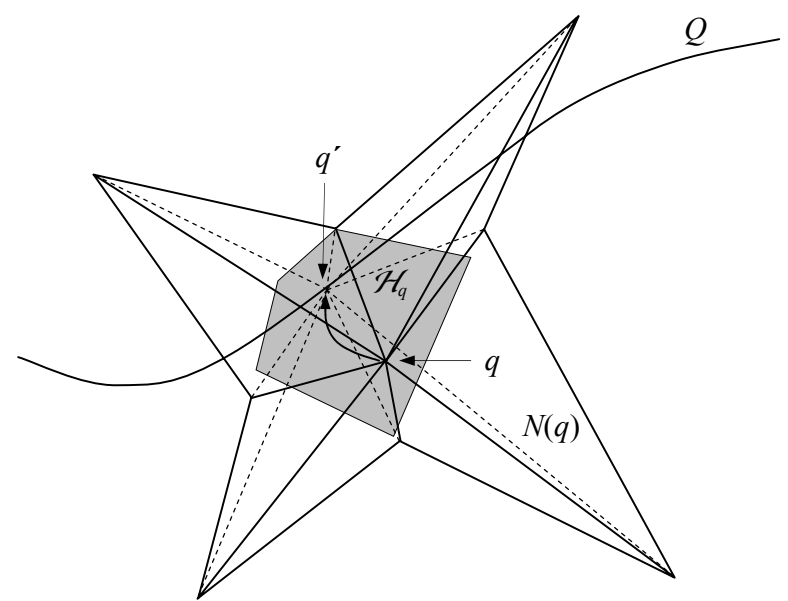

Fig. 2 The curve $Q$ intersects the feasible region $\mathscr{H}_{q}$ (in gray) and, therefore, the node $q$ is projectable, being $q^{\prime}$ its optimal position on the curve

When $\Sigma$ is a curved surface, each triangle of $M(p)$ is placed on a different plane. Therefore, it is not possible to define the feasible region associated to the free node $p$. Nevertheless, the feasible region associated to node $q$ is perfectly defined in plane $P$ and it is denoted as $\mathscr{H}_{q}$. Furthermore, its associated objective function has a barrier at the boundary of $\mathscr{H}_{q}$ (see [6]). This is a crucial reason for working on $P$ instead of on $\Sigma$.

In the present work the curve $C$ is defined as the image of a curve $Q$ sited on a plane $P$. Specifically, if we define a plane curve by the parametrization $Q(u)=(x(u), y(u))$ and we consider that $f(x, y)$ is the $z$ coordinate of the underlaying surface (the true surface, if it is known, or the piecewise linear interpolation, if it is not), then the curve $C$ is given by $C(u)=(x(u), y(u), f(x(u), y(u)))$ (see Figure 3 ). We note that this type of parametrization can be straight- 
forward introduced in the new meccano method which has been recently developed by the authors [4]. We remark also that, although the surface mesh smoothing process can be carried out in different planes chosen in terms of $M(p)$ [5], the particular way in which $C$ is defined in the present paper demands a unique plane. A general parametric curve $C(u)=(x(u), y(u), z(u))$ will be considered in future works.

Since the problem of getting a piecewise approximation of $C$ by edges of $T_{\Sigma}$ is translated to the plane $P$, the task to determine if a node $q$ can be projected onto $Q$ and, that being the case, which is its optimal position, is undertaken by an objective function derived from algebraic quality measures of the local mesh $N(q)$. This objective function incorporates the modifications proposed in [6] in order to deal with tangled meshes. Obviously, a control of the allowed distance between $M(p)$ and $M\left(p^{\prime}\right)$ is done in the analysis of the movement of node $q$.

\section{Smoothing and Untangling of Plane Triangulations}

Firstly, we will focus our attention on finding an objective function to smooth a valid plane triangulation. As it is shown in [9], [14], and [15] we can derive optimization functions from algebraic quality measures of the elements belonging to a local mesh. Let us consider a triangular mesh $T_{P}$ defined in $\mathbb{R}^{2}$ and let $t$ be a triangle in the physical space whose vertices are given by $\mathbf{x}_{k}=\left(x_{k}, y_{k}\right)^{T} \in \mathbb{R}^{2}, k=0,1,2$. To start with, we introduce an algebraic quality measure for $t$. Let $t_{R}$ be the reference triangle with vertices $\mathbf{u}_{0}=(0,0)^{T}, \mathbf{u}_{1}=$ $(1,0)^{T}$, and $\mathbf{u}_{2}=(0,1)^{T}$. If we choose $\mathbf{x}_{0}$ as the translation vector, the affine map that takes $t_{R}$ to $t$ is $\mathbf{x}=A \mathbf{u}+\mathbf{x}_{0}$, where $A$ is the Jacobian matrix of the affine map referenced to node $\mathbf{x}_{0}$, given by $A=\left(\mathbf{x}_{1}-\mathbf{x}_{0}, \mathbf{x}_{2}-\mathbf{x}_{0}\right)$. We will denote this type of affine maps as $t_{R} \stackrel{A}{\rightarrow} t$. Let now $t_{I}$ be an ideal triangle whose vertices are $\mathbf{w}_{k} \in \mathbb{R}^{2},(k=0,1,2)$ and let $W_{I}=\left(\mathbf{w}_{1}-\right.$ $\left.\mathbf{w}_{0}, \mathbf{w}_{2}-\mathbf{w}_{0}\right)$ be the Jacobian matrix, referenced to node $\mathbf{w}_{0}$, of the affine map $t_{R} \stackrel{W_{I}}{\rightarrow} t_{I}$; then, we define $S=A W_{I}^{-1}$ as the weighted Jacobian matrix of the affine map $t_{I} \stackrel{S}{\rightarrow} t$. In the particular case that $t_{I}$ was the equilateral triangle $t_{E}$, the Jacobian matrix $W_{I}=W_{E}$ will be defined by $\mathbf{w}_{0}=(0,0)^{T}$, $\mathbf{w}_{1}=(1,0)^{T}$ and $\mathbf{w}_{2}=(1 / 2, \sqrt{3} / 2)^{T}$.

We can use matrix norms, determinant or trace of $S$ to construct algebraic quality measures of $t$. For example, the Frobenius norm of $S$, defined by $|S|=\sqrt{\operatorname{tr}\left(S^{T} S\right)}$, is specially indicated because it is easily computable. Thus, it is shown in [16] that $q_{\eta}=\frac{2 \sigma}{|S|^{2}}$ is an algebraic quality measure of $t$, where $\sigma=\operatorname{det}(S)$. We use this quality measure to construct an objective function. Let $\mathbf{x}=(x, y)^{T}$ be the position vector of the free node $q$, and let $S_{m}$ be the weighted Jacobian matrix of the $m$-th triangle of a valid local mesh $N(q)$ composed of $M$ triangles, see Figure 2. The objective function associated to $m$-th triangle is $\frac{\left|S_{m}\right|^{2}}{2 \sigma_{m}}$, and the corresponding objective function for the local mesh is

$\left|K_{\eta}\right|_{n}(\mathbf{x})=\left[\sum_{m=1}^{M}\left(\frac{\left|S_{m}\right|^{2}}{2 \sigma_{m}}\right)^{n}(\mathbf{x})\right]^{\frac{1}{n}}$

being $n$ an integer number, typically $n=1$ or $n=2$.

The feasible region for the local mesh is defined as the interior of the polygonal set $\mathscr{H}_{q}=\bigcap_{m=1}^{M} H_{m}$, where $H_{m}$ are the half-planes defined by $\sigma_{m}(\mathbf{x}) \geq 0$. We say that a triangle is inverted if $\sigma<0$. The objective function (1) presents a barrier in the boundary of the feasible region. This barrier avoids the optimization method to create a tangled mesh when it starts with a valid one, but, on the other hand, it prevents the algorithm to untangle it when there are inverted elements. Therefore, this objective function is only appropriate to improve the quality of a valid mesh, not to untangle it. To construct an objective function applicable to deal with tangled meshes we propose to modify it following the criteria developed in [6]. This modification lies in substituting $\sigma$ in (1) by the positive and increasing function

$h(\sigma)=\frac{1}{2}\left(\sigma+\sqrt{\sigma^{2}+4 \delta^{2}}\right)$

where the parameter $\delta=h(0)$ is an appropriate small value.

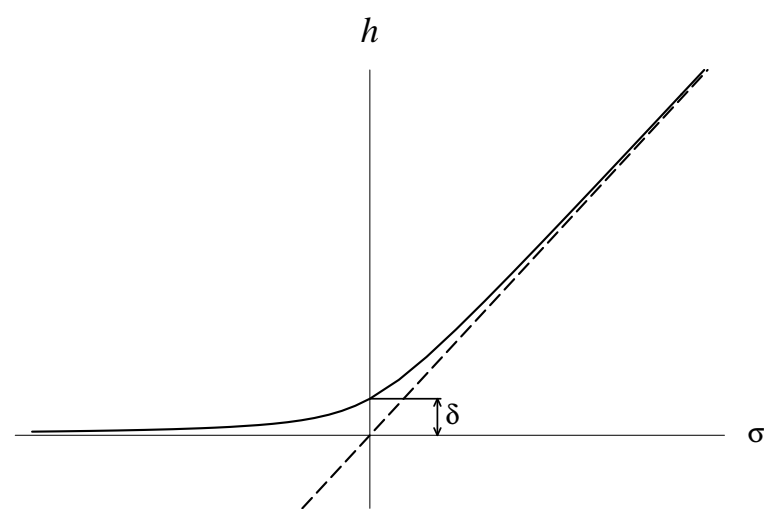

Fig. 3 Representation of function $h(\sigma)$.

The behavior of $h(\sigma)$ in function of $\delta$ parameter is such that, $\lim _{\delta \rightarrow 0} h(\sigma)=\sigma, \forall \sigma \geq 0$ and $\lim _{\delta \rightarrow 0} h(\sigma)=0, \forall \sigma \leq 0$. Thus, if int $\mathscr{H}_{q} \neq \emptyset$, then $\forall \mathbf{x} \in$ int $\mathscr{H}_{q}$ we have $\sigma_{m}(\mathbf{x})>0$, for $m=$ $1,2, \ldots, M$ and, as smaller values of $\delta$ are chosen, $h\left(\sigma_{m}\right)$ behaves very much as $\sigma_{m}$, so that, the original objective function and its corresponding modified version are very close in the feasible region.

In this way, the barrier associated with the singularities of $\left|K_{\eta}\right|_{n}(\mathbf{x})$ will be eliminated and the modified objective function will be smooth all over $\mathbb{R}^{2}$ 
$\left|K_{\eta}^{\prime}\right|_{n}(\mathbf{x})=\left[\sum_{m=1}^{M}\left(\frac{\left|S_{m}\right|^{2}}{2 h\left(\sigma_{m}\right)}\right)^{n}(\mathbf{x})\right]^{\frac{1}{n}}$

This new objective function strongly penalizes the negative values of $\sigma$, so that the minimization process of (3) leads to the construction of a local mesh $N\left(q^{\prime}\right)$ without inverted triangles, provided it is possible. Note that the minimum of original and modified functions are nearly identical when $\mathscr{H}_{q} \neq \emptyset$ and $\delta$ tends to zero. With this approach, we can use any standard and efficient unconstrained optimization method to find the minimum of the modified objective function, see for example [2].

\section{Alignment of Plane Triangulations}

Node movement provides the mesh with the ability to align with an arbitrary curve. Suppose that $Q$ is a curve defined on a 2-D triangulation $T_{P}$, our objective is to move some nodes of $T_{P}$, projecting them onto $Q$, to get an interpolation of $Q$ by linked edges of $T_{P}$. To achieve this objective we have to decide which nodes of $T_{P}$ can be projected onto $Q$ without inverting any triangle of its local mesh. More accurately, we say that the free node $q$ is projectable onto $Q$ if there are points of this curve belonging to the feasible region $\mathscr{H}_{q}$ (see Figure 2).

In general, if $q$ is projectable, its possible placement on $Q$ is not unique. The projecting/smoothing method must determine if $q$ can be projected onto $Q$ and, if so, which is its optimal position. The last question can be answered by using the objective function (3) subject to the constrained $\mathbf{x} \in Q$. Thus, the problem of finding the optimal position to project the free node onto the curve is

$\operatorname{minimize}\left|K_{\eta}^{\prime}\right|_{n}(\mathbf{x})$, subject to $\mathbf{x} \in Q$

If $\overline{\mathbf{x}}$ is the position vector of the minimizing point $q^{\prime}$ of (4) and $\sigma(\overline{\mathbf{x}})>0$ for all triangle of $N\left(q^{\prime}\right)$, we conclude that $q$ is projectable onto $Q$ and $\overline{\mathbf{x}}$ is its optimal position. Otherwise, we say that node $q$ is not projectable.

\subsection{Curve Definition}

The previous criterion allows us to determine whether $q$ is projectable onto $Q$ or not, but it involves a high computational cost because it needs to solve the constrained minimization problem (4). Nevertheless, it is clear that most nodes of $T_{P}$ are not projectable because they are very far from any point of the curve. Therefore, it is convenient to have an efficient method to select those nodes, close to some segment of $Q$, expected to be projectable.
In many situations of practical interest we do not have an analytical representation of $Q$, but $Q$ is approximately known by a sequence of interpolating data points. Among the options to define an interpolating curve, we have chosen a parametric cubic spline as it has many desired properties: it is a $C^{2}$ continuous function, it has a very simple local form, small oscillations, etc. Moreover, each segment of the spline is a degree 3 Bézier curve that lies within the convex hull of its four defining control points (see, for example [1]). We will use this property in order to know if a given node is close to some segment of $Q$.

Let $\left\{P_{0}, P_{1}, \ldots, P_{m}\right\} \subset \mathbb{R}^{2}$ be a set of interpolating points belonging to plane $P$. The parametric cubic spline

$Q(u)=(x(u), y(u))$, where $u \in\left[u_{0}, u_{m}\right]$

is an interpolating curve that satisfies $Q\left(u_{i}\right)=P_{i}$ for $i=$ $0, \ldots, m$ and two additional constraints in order to be fully defined. Usually, these constraints are imposed at the ends of the curve. For example, it is well known that the conditions $Q^{\prime \prime}\left(u_{0}\right)=0$ and $Q^{\prime \prime}\left(u_{m}\right)=0$ define a spline known as natural.

Every segment of the spline delimited by two consecutive interpolating points is a degree 3 polynomial. Suppose that $Q_{i}(t)=\mathbf{a}^{i}+\mathbf{b}^{i} t+\mathbf{c}^{i} t^{2}+\mathbf{d}^{i} t^{3}$, with $\mathbf{a}^{i}, \mathbf{b}^{i}, \mathbf{c}^{i}$ and $\mathbf{d}^{i}$ in $\mathbb{R}^{2}$, is the polynomial associated to the segment $Q_{i}$ $(i=0,1, \ldots, m-1)$ that runs from $P_{i}$ to $P_{i+1}$, being $t \in[0,1]$ the local parameter, see Figure 4. This one is related with the parameter of the entire curve by $t=\left(u-u_{i}\right) /\left(u_{i+1}-u_{i}\right)$.

\subsection{Node Projection onto the Curve}

The $Q_{i}$ segment also is a degree 3 Bézier curve, given by $Q_{i}(t)=\sum_{j=0}^{3} \mathbf{u}_{j}^{i} B_{j}^{i}(t)$ with $t \in[0,1]$, where $B_{j}^{i}(t)$ are the Berstein polynomials and $\mathbf{u}_{j}^{i} \in \mathbb{R}^{2}$ are the control points. The relation between the polynomial coefficients and the control points are given by

$$
\left(\begin{array}{l}
\mathbf{u}_{0}^{i} \\
\mathbf{u}_{1}^{i} \\
\mathbf{u}_{2}^{i} \\
\mathbf{u}_{3}^{i}
\end{array}\right)=\frac{1}{3}\left(\begin{array}{llll}
3 & 0 & 0 & 0 \\
3 & 1 & 0 & 0 \\
3 & 2 & 1 & 0 \\
3 & 3 & 3 & 3
\end{array}\right)\left(\begin{array}{c}
\mathbf{a}^{i} \\
\mathbf{b}^{i} \\
\mathbf{c}^{i} \\
\mathbf{d}^{i}
\end{array}\right)
$$

As we said, an interesting property of the Bézier curves establishes that the $Q_{i}$ segment lies within the convex hull of its control points. If $\mathrm{CH}$ denotes the convex hull of a set of points, we have $Q_{i} \subseteq \mathrm{CH}\left(\mathbf{u}_{0}^{i}, \ldots, \mathbf{u}_{3}^{i}\right)$. Note that a necessary (but not sufficient) condition for the node $q$ to be projectable onto $Q$ is that its feasible region $\mathscr{H}_{q}$ intersects the convex hull of some segment of the curve. In other words, it must exist a segment $Q_{i}$ such that $\mathscr{H}_{q} \cap \mathrm{CH}\left(\mathbf{u}_{0}^{i}, \ldots, \mathbf{u}_{3}^{i}\right) \neq \emptyset$. This property allows us to know beforehand which nodes are not 
projectable, because they yield an empty intersection for all segments of the curve. Nevertheless, calculating the set $\mathscr{H}_{q}$ and, moreover, its intersection with a convex set, is not a trivial problem, so it is more advisable to deal with a simplified version.

Let $R_{q}$ and $R_{Q_{i}}$ be the minimal rectangles, with sides parallel to the axes, enclosing the sets $N(q)$ and $\mathrm{CH}\left(\mathbf{u}_{0}^{i}, \ldots, \mathbf{u}_{3}^{i}\right)$, respectively. Then, due to $\mathscr{H}_{q} \subset R_{q}$, it is clear that $q$ is projectable onto $Q_{i}$ only if $R_{q} \cap R_{Q_{i}} \neq \emptyset$ (see Figure 4). The computation of this intersection allows us to take a quick decision about if a node is candidate to be projected onto the curve.

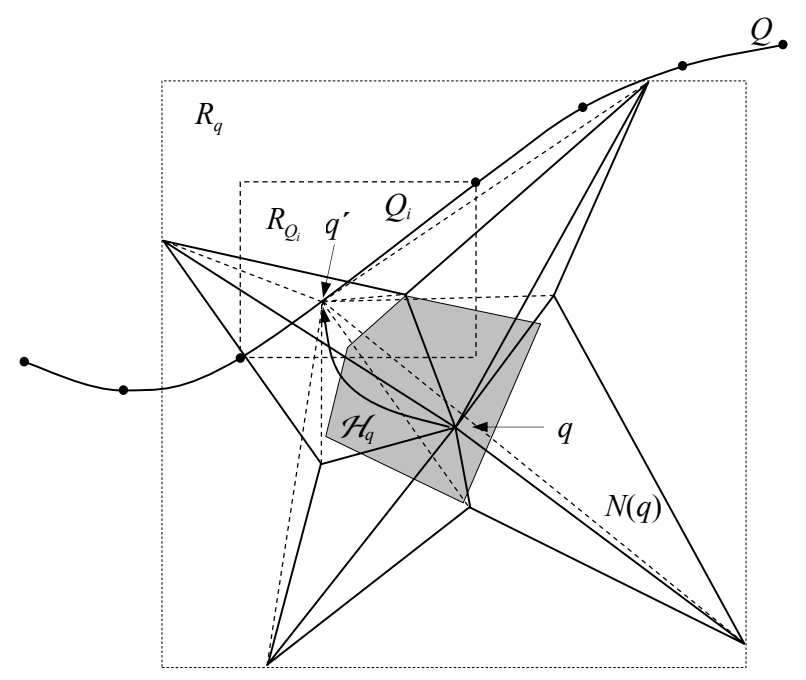

Fig. 4 The figure shows the situation in which $R_{q} \cap R_{Q_{i}} \neq \emptyset$, but node $q$ is not projectable because the optimal position for the free node, $q^{\prime}$, is outside the feasible region

The algorithm to determine if $q$ is projectable onto $Q$ and, if it is so, which is its optimal position, can be summarized as follows. For each segment of the curve analyze $R_{q} \cap R_{Q_{i}}$ and, if this set is not empty, solve the minimization problem

$\operatorname{minimize}\left|K_{\eta}^{\prime}\right|_{n}\left(Q_{i}(t)\right)$, for $t \in[0,1]$

Let $\bar{t}$ be the global minimum of (7) and $\overline{\mathbf{x}}_{i}=Q_{i}(\bar{t})$ the corresponding position of the free node $q$ on the segment $Q_{i}$. We say that $\overline{\mathbf{x}}_{i}$ is an admissible optimal position for the free node if $\sigma_{m}\left(\overline{\mathbf{x}}_{i}\right)>0$ for $m=1, \ldots, M$. In order to determine the optimal position of the free node on $Q$, we take $\overline{\mathbf{x}}_{\text {opt }}$ as the best admissible position for all segments $Q_{i}$. Obviously, if no admissible position exists, the conclusion of previous algorithm is that node $q$ is not projectable onto $Q$.

Note that an admissible projection of a free node on $Q$ can give rise to a local mesh with very poor quality. Although this effect is partly palliated after smoothing the re- mainder nodes, following the procedure described in section 3 , it is better to tighten the condition $\sigma_{m}\left(\overline{\mathbf{x}}_{i}\right)>0$ enforcing $\sigma_{m}\left(\overline{\mathbf{x}}_{i}\right)>\varepsilon$, with $\varepsilon>0$ a prescribed tolerance. Nevertheless, this more restrictive condition makes it difficult for the nodes to be projected onto the curve and it could produce situations where some sections of the curve are not interpolated by edges of $T_{P}$. This drawback will be studied in the next subsection but, for that purpose, it needs further clarification.

Up to now, we have accepted that parameter $t$ pertains to the closed interval $[0,1]$ and, in consequence, the problem (7) admits a global minimum. But, with this consideration, the ends of the consecutive segments are shared and, therefore, a projected point can belong to two segments at the same time. In order to avoid this ambiguity, we will assume that each segment $Q_{i}(t)$ is defined for $t \in[0,1)$, except the last one, that it is for $t \in[0,1]$ if the curve is open. In this way, each point of the curve belongs to a unique segment.

\subsection{Discontinuities of the Mesh Alignment with the Curve}

It can happen that, after repositioning all the nodes of the mesh, the piecewise approximation of $Q$ by edges of $T_{P}$ is not continuous. We can detect this discontinuity if we take into account that the projected nodes are arranged in the curve. Thus, a section of the interpolated curve among two consecutive projected nodes is discontinuous if they are not connected by an edge of $T_{P}$.

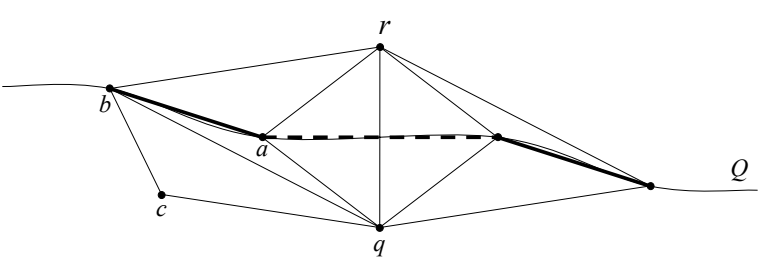

(a)

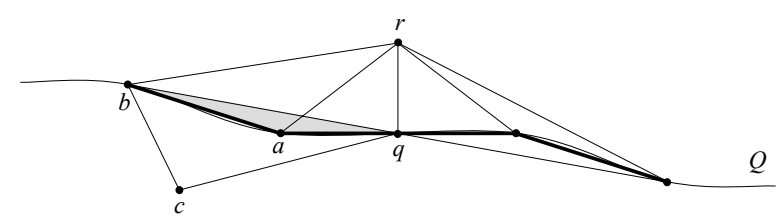

(b)

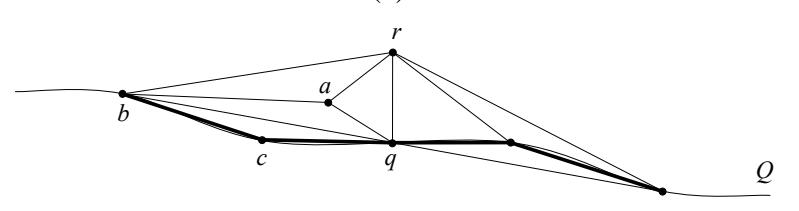

(c)

Fig. 5 The dashed line is non-recoverable without tangling the mesh (a). The free node $q$ is enforced to be projected (b). The tangled triangle $a b q$ is untangled and the node $c$ is also projected (c) 
As the parameter $t \in[0,1)$ induces an order relation in each segment of the curve and, in turn, each segment is ordered by its subindex, we can say that the node $p \in Q_{i}$ precedes $p^{\prime} \in Q_{j}$ if $i<j$ or, in case of $i=j$, if the corresponding parameters satisfies $t_{p}<t_{p^{\prime}}$. A possibility to correct a detected discontinuity in the piecewise approximation of $Q$ is to relax the condition $\sigma_{m}\left(\overline{\mathbf{x}}_{i}\right)>\varepsilon$, by decreasing the value of $\varepsilon$. However, there are situations in which, even taking $\varepsilon$ equal to zero, there are discontinuities impossible to avoid without removing some of the projected nodes. The Figure 5(a) shows a scheme of this problem. It can be seen that it is impossible to project the node $q$ (neither $r$ ) without tangling the mesh. We propose a solution to this conflict by enforcing the free node $q$ to be projected, even if a tangled mesh is created. The Figure 5(b) shows how the movement of $q$ produces the tangled triangle $a b q$.

Afterward, the position of $q$ is fixed for subsequent iterations of the projecting/smoothing algorithm, but the surrounding nodes are free to move in search of their optimal positions that untangle the mesh and complete the interpolation of the curve (see 5(c)). So, the algorithm extracts nodes from the curve if their current positions are not admissible (see the new position of node $a$ in figure 5(c)).

Sometimes the curve represented by splines has sharp features that we want to preserve in the piecewise interpolation. To reach this objective we select, from the interpolating points, a set of prescribed points sited in strategic locations. Once the projecting/smoothing process has finished, the algorithm searches among the nodes projected on $Q$, which one is the optimal candidate, say $q$, to be relocated in the position of each prescribed node. If $\mathbf{x}_{\text {pres }}$ is the position of certain prescribed point, the node $q$ is chosen, among the nodes projected on $Q$ and close to $\mathbf{x}_{\text {pres }}$, as the one that maximizes the quality of $N(q)$ when $q$ is enforced to take the position $\mathbf{x}_{\text {pres }}$. Obviously, if $N(q)$ is not valid after the relocation of $q$, a new iteration of the projecting/smoothing procedure must be done, keeping fixed the position of $q$ in $\mathbf{x}_{\text {pres. }}$.

\section{Extension to Curved Surfaces}

We are interested in extending the projecting/smoothing method to curved surfaces. As we pointed in section 2, the original problem on $\Sigma$ is transformed into another one on the plane $P$, where the projecting/smoothing process is performed. The more significant difference with respect to the former method lies in searching ideal triangles in $N(q)$ that become equilateral in $M(p)$. This question was studied in [5] with regard to smoothing of surface triangular meshes. In order to be auto-consistent, we have considered appropriate to present in subsection 5.1 the main results of this work. Its connection with the problem of mesh aligning with curves will be introduced in subsection 5.2.
5.1 Similarity Transformation for Surface and Parametric Triangulations

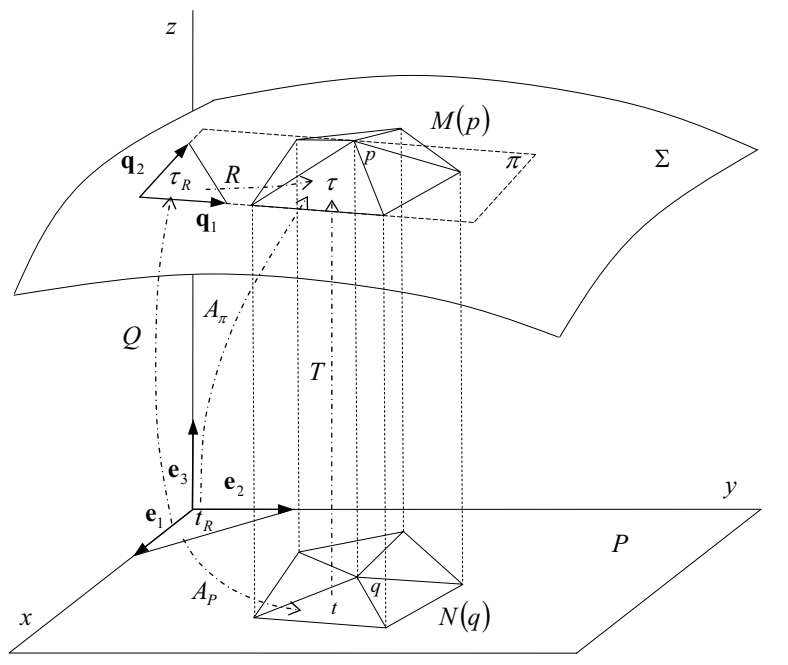

Fig. 6 Local surface mesh, $M(p)$, and its projection, $N(q)$, on plane $P$

Suppose that for each local mesh $M(p)$ placed on the surface $\Sigma$, that is, with all its nodes on $\Sigma$, it is possible to find a plane $P$ such that the orthogonal projection of $M(p)$ on $P$ is a valid mesh $N(q)$. In present work we assume that the plane is the same for all the local meshes involved in the interpolation of $C$. Suppose, as well, that we define the axes in such a way that the $x, y$-plane coincide with $P$. If, at least in $\mathscr{H}_{q}$, it is possible to define the surface $\Sigma$ by the parametrization $\mathbf{s}(x, y)=(x, y, f(x, y))$, where $f$ is a continuous function, then, we can optimize $M(p)$ by an appropriate optimization of $N(q)$. We will refer to $N(q)$ as the parametric mesh. The basic idea consists of finding the position of $q$ in $\mathscr{H}_{q}$ that makes $M(p)$ be an optimum local mesh. To do this, we search ideal elements in $N(q)$ that become equilateral in $M(p)$.

Let $\tau \in M(p)$ be a triangular element on $\Sigma$ whose vertices are given by $\mathbf{y}_{k}=\left(x_{k}, y_{k}, z_{k}\right)^{T},(k=0,1,2)$ and $t_{R}$ be the reference triangle in $P$ (see Fig. 6). If we choose $\mathbf{y}_{0}$ as the translation vector, the affine map $t_{R} \stackrel{A_{\pi}}{\rightarrow} \tau$ is $\mathbf{y}=A_{\pi} \mathbf{u}+\mathbf{y}_{0}$, where $\mathbf{u}$ is the vector of a generic point of $t_{R}$ and $A_{\pi}$ is its Jacobian matrix, given by $A_{\pi}=\left(\mathbf{y}_{1}-\mathbf{y}_{0}, \mathbf{y}_{2}-\mathbf{y}_{0}\right)$.

Now, consider that $t \in N(q)$ is the orthogonal projection of $\tau$ on $P$. Then, the vertices of $t$ are $\mathbf{x}_{k}=\Pi \mathbf{y}_{k}=\left(x_{k}, y_{k}\right)^{T}$, $(k=0,1,2)$, where $\Pi=\left(\mathbf{e}_{1}, \mathbf{e}_{2}\right)^{T}$ is $2 \times 3$ matrix of the affine map $\tau \stackrel{\Pi}{\rightarrow} t$, being $\left\{\mathbf{e}_{1}, \mathbf{e}_{2}, \mathbf{e}_{3}\right\}$ the canonical basis in $\mathbb{R}^{3}$ (the associated projector from $\mathbb{R}^{3}$ to $P$, considered as a subspace of $\mathbb{R}^{3}$, is $\Pi^{T} \Pi$ ). Taking $\mathbf{x}_{0}$ as translation vector, the affine $\operatorname{map} t_{R} \stackrel{A_{P}}{\rightarrow} t$ is $\mathbf{x}=A_{P} \mathbf{u}+\mathbf{x}_{0}$, where $A_{P}=\Pi A_{\pi}$ is its Jacobian matrix $A_{P}=\left(\mathbf{x}_{1}-\mathbf{x}_{0}, \mathbf{x}_{2}-\mathbf{x}_{0}\right)$. 
Therefore, the $3 \times 2$ matrix of the affine map $t \stackrel{T}{\rightarrow} \tau$ is

$T=A_{\pi} A_{P}^{-1}$

Let $V_{\pi}$ be the subspace spanned by the column vectors of $A_{\pi}$ and let $\pi$ be the plane defined by $V_{\pi}$ and the point $\mathbf{y}_{0}$. Our goal is to find the ideal triangle $t_{I} \subset P$, moving $q$ on $P$, such that $t_{I}$ is mapped by $T$ into an equilateral one, $\tau_{E} \subset \pi$. In general, the strict fulfillment of this requirement is only possible if $N(q)$ is formed by a unique triangle.

Due to $\operatorname{rank}\left(A_{\pi}\right)=\operatorname{rank}\left(A_{P}\right)=2$, it exists a unique factorization $A_{\pi}=Q R$, where $Q$ is an orthogonal matrix $\left(Q^{T} Q=\right.$ $I)$ and $R$ is an upper triangular one with $[R]_{i i}>0(i=1,2)$. The columns of the $3 \times 2$ matrix $Q$ define an orthonormal basis $\left\{q_{1}, q_{2}\right\}$ that spans $V_{\pi}$, so we can see $Q$ as the matrix of the affine map $t_{R} \stackrel{Q}{\rightarrow} \tau_{R}$ and $R$ as the $2 \times 2$ Jacobian matrix of the affine map $\tau_{R} \stackrel{R}{\rightarrow} \tau$ (see Fig. 6). As $t_{R} \stackrel{W_{E}}{\longrightarrow} t_{E}$ and $Q$ is an orthogonal matrix that keeps the angles and norms of the vectors, then $t_{E} \stackrel{Q}{\rightarrow} \tau_{E}$ and, therefore

$Q W_{E}=A_{\pi} R^{-1} W_{E}$

is the $3 \times 2$ Jacobian matrix of affine map $t_{R} \stackrel{Q W_{E}}{\rightarrow} \tau_{E}$. On the other hand, we define on the plane $\pi$

$S=R W_{E}^{-1}$

as the $2 \times 2$ weighted Jacobian matrix of the affine map that transforms the equilateral triangle into the physical one, that is, $\tau_{E} \stackrel{S}{\rightarrow} \tau$.

We have chosen as ideal triangle in $\pi$ the equilateral one $\left(\tau_{I}=\tau_{E}\right)$, then, the Jacobian matrix $W_{I}$ of the affine map $t_{R} \stackrel{W_{I}}{\rightarrow} t_{I}$ is calculated by imposing the condition $T W_{I}=Q W_{E}$, because $t_{R} \stackrel{T W_{I}}{\longrightarrow} \tau_{I}$ and $t_{R} \stackrel{Q W_{E}}{\longrightarrow} \tau_{E}$. Taking into account (9), it yields

$T W_{I}=A_{\pi} R^{-1} W_{E}$

and, from (8), we obtain

$W_{I}=A_{P} R^{-1} W_{E}$

so we define on $P$ the ideal-weighted Jacobian matrix of the affine map $t_{I} \stackrel{S_{I}}{\rightarrow} t$ as $S_{I}=A_{P} W_{I}^{-1}$. From (12) it results

$S_{I}=A_{P} W_{E}^{-1} R A_{P}^{-1}$

and, from (10)
$S_{I}=A_{P} W_{E}^{-1} S W_{E} A_{P}^{-1}=S_{E} S S_{E}^{-1}$

where $S_{E}=A_{P} W_{E}^{-1}$ is the equilateral-weighted Jacobian matrix of the affine map $t_{E} \stackrel{S_{E}}{\longrightarrow} t$. Finally, from (3), we obtain the next similarity transformation.

$S=S_{E}^{-1} S_{I} S_{E}$

Therefore, it can be said that the matrices $S$ and $S_{I}$ are similar.

\subsection{Projecting and Smoothing on the Parametric Space}

Matrix S, as it is defined in (15), might be used to construct the objective function associated to $M(p)$ and, then, solve the optimization problem. Nevertheless, this procedure has important disadvantages. First, the optimization of $M(p)$, working on the true surface, would require the imposition of the constraint $p \in \Sigma$. It would complicate the resolution of the problem because, in many cases, $\Sigma$ is not defined by a smooth function. Moreover, when the local mesh $M(p)$ is on a curved surface, each triangle is sited on a different plane and the objective function, constructed from $S$, lacks barriers. It is impossible to define a feasible region in the same way as it was done at section 3 . Indeed, all the positions of the free node, except those that make $\operatorname{det}(S)=0$ for any triangle, produce correct triangulations of $M(p)$. However, for many purposes as, for example, to construct a 3-D mesh from the surface triangulation, there are unacceptable positions of the free node.

To overcome these difficulties we propose to carry out the optimization of $M(p)$ in an indirect way, working on $N(q)$. With this approach the movement of the free node will be restricted to $\mathscr{H}_{q}$, which avoids unacceptable surface triangulations to be formed.

Let us consider that $\mathbf{x}=(x, y)^{T}$ is the position vector of the free node $q$, sited on the plane $P$. If we suppose that $\Sigma$ is parametrized by $\mathbf{s}(x, y)=(x, y, f(x, y))$, then, the position of the free node $p$ on the surface is given by $\mathbf{y}=$ $(x, y, f(x, y))^{T}=(\mathbf{x}, f(\mathbf{x}))^{T}$.

Note that $S_{E}=A_{P} W_{E}^{-1}$ only depends on $\mathbf{x}$ because $W_{E}$ is constant and $A_{P}$ is a function of $\mathbf{x}$. Besides, $S_{I}=A_{P} W_{I}^{-1}$ depends on $\mathbf{y}$, due to $W_{I}=A_{P} R^{-1} W_{E}$, and $R$ is a function of $\mathbf{y}$. Thus, we have $S_{E}(\mathbf{x})$ and $S_{I}(\mathbf{y})$. We shall optimize the local mesh $M(p)$ by an iterative procedure maintaining constant $W_{I}(\mathbf{y})$ in each step. To do this, at the first step, we fix $W_{I}(\mathbf{y})$ to its initial value, $W_{I}^{0}=W_{I}\left(\mathbf{y}^{0}\right)$, where $\mathbf{y}^{0}$ is given by the initial position of $p$. So, if we define $S_{I}^{0}(\mathbf{x})=A_{P}(\mathbf{x})\left(W_{I}^{0}\right)^{-1}$, we approximate the similarity transformation (15) as

$S^{0}(\mathbf{x})=S_{E}^{-1}(\mathbf{x}) S_{I}^{0}(\mathbf{x}) S_{E}(\mathbf{x})$ 
Now, the construction of the objective function is carried out in a standard way following equation (1), but using $S^{0}$ instead of $S$. So, the objective function for a given triangle $\tau \subset \pi$ is $\frac{\left|S^{0}(\mathbf{x})\right|^{2}}{2 \sigma^{0}(\mathbf{x})}$, where $\sigma^{0}(\mathbf{x})=\operatorname{det}\left(S^{0}(\mathbf{x})\right)$.

With this approach the optimization of the local mesh $M(p)$ is transformed into a two-dimensional problem without constraints, defined on $N(q)$, and, therefore, it can be solved with low computational cost. Furthermore, if we write $W_{I}^{0}$ as $A_{P}^{0}\left(R^{0}\right)^{-1} W_{E}$, where $A_{P}^{0}=A_{P}\left(\mathbf{x}^{0}\right)$ and $R^{0}=R\left(\mathbf{y}^{0}\right)$, it is straightforward to show that $S^{0}$ can be simplified as

$S^{0}(\mathbf{x})=R^{0}\left(A_{P}^{0}\right)^{-1} S_{E}(\mathbf{x})$

and our objective function for the local mesh is

$\left|K_{\eta}^{0}\right|_{n}(\mathbf{x})=\left[\sum_{m=1}^{M}\left(\frac{\left|S_{m}^{0}\right|^{2}}{2 \sigma_{m}^{0}}\right)^{n}(\mathbf{x})\right]^{\frac{1}{n}}$

Let now analyze the behavior of the objective function when the free node crosses the boundary of the feasible region. Denoting $\alpha_{P}=\operatorname{det}\left(A_{P}\right), \alpha_{P}^{0}=\operatorname{det}\left(A_{P}^{0}\right), \rho^{0}=\operatorname{det}\left(R^{0}\right)$, $\omega_{E}=\operatorname{det}\left(W_{E}\right)$ and taking into account (17), we can write

$\sigma^{0}=\rho^{0}\left(\alpha_{P}^{0}\right)^{-1} \alpha_{P} \omega_{E}^{-1}$

Note that $\rho^{0}, \alpha_{P}^{0}$ and $\omega_{E}$ are constants different of zero when the initial mesh is valid, so the singularities of (18) happens when $\alpha_{P}=0$, that is, when $q$ is placed on the boundary of $\mathscr{H}_{q}$. This singularity determines a barrier in the objective function that prevents the optimization algorithm to take the free node outside this region. Remark that this barrier does not appear if we use the exact weighted Jacobian matrix $S$, given in (15), due to $\operatorname{det}(R)=R_{11} R_{22}>0$.

We can reason as in section 3 to justify that objective function (18) is appropriate for smoothing but not for untangling. The goal is to regularize the objective function following a similar procedure as described in section 3 . Note that when the mesh is tangled, they can appear degenerated triangles with $\alpha_{P}^{0}=0$ and, therefore, the matrix $A_{P}^{0}$ becomes singular. This singularity can be avoided by using the function $h(\sigma)$, see equation (2), and taking into account that $\left(A_{P}^{0}\right)^{-1}$ can be written as $\Delta_{P}^{0} / \alpha_{P}^{0}$, where $\Delta_{P}^{0}$ is the adjoint matrix of $A_{P}^{0}$. Therefore, attending to (17) and (19), we can write the terms of (18) as

$\frac{\left|S^{0}\right|^{2}}{2 \sigma^{0}}=\frac{\left|R^{0} \Delta_{P}^{0} S_{E}\right|^{2}}{2\left(\alpha_{P}^{0}\right)^{2} \sigma^{0}}=\frac{\left|R^{0} \Delta_{P}^{0} S_{E}\right|^{2}}{2 \rho^{0} \alpha_{P}^{0} \alpha_{P} \omega_{E}^{-1}}$

Finally, if we write $\hat{S}^{0}=R^{0} \Delta_{P}^{0} S_{E}$ and $\hat{\sigma}^{0}=\rho^{0} \alpha_{P}^{0} \alpha_{P} \omega_{E}^{-1}$, the regularized objective function able to untangle the mesh is
$\left|K_{\eta}^{0^{\prime}}\right|_{n}(\mathbf{x})=\left[\sum_{m=1}^{M}\left(\frac{\left|\hat{S}_{m}^{0}\right|^{2}}{2 h\left(\hat{\sigma}_{m}^{0}\right)}\right)^{n}(\mathbf{x})\right]^{\frac{1}{n}}$

We have transformed the original smoothing problem in a two-dimensional approach on $P$, due to (21) is a function of $x$ and $y$ coordinates, exclusively. The algorithm to determine the optimal projection of a free node $p \in \Sigma$ onto the curve $C$ is similar to the one described in section 4.2, but using $\left|K_{\eta}^{0^{\prime}}\right|_{n}$ given in (21) as objective function of the minimization problem (7). If the optimal projection of the free node $q$ onto $Q$ is $\overline{\mathbf{x}}_{o p t}$, then, the corresponding position on the surface is given by $\overline{\mathbf{y}}_{\text {opt }}=\left(\overline{\mathbf{x}}_{\text {opt }}, f\left(\overline{\mathbf{x}}_{\text {opt }}\right)\right)^{T}$, where $f(\mathbf{x})$ is the $z$ coordinate of the underlaying surface. If this one is not known analytically, we take the initial triangulation as reference surface. The algorithm follows the usual smoothing procedure when the free node is not projectable on $C$. The discontinuities of the mesh alignment with the curve are solved by using the same idea of section 4.3.

Suppose that $\mathbf{x}^{1}=\overline{\mathbf{x}}^{0}$ is the minimizing point. As the objective function has been constructed by keeping $\mathbf{y}$ in its initial position, $\mathbf{y}^{0}$, then $\mathbf{x}^{1}$ is only the first approximation to our problem. This result is improved updating the objective function at $\mathbf{y}^{1}=\left(\mathbf{x}^{1}, f\left(\mathbf{x}^{1}\right)\right)^{T}$ and, then, computing the new minimizing position, $\mathbf{x}^{2}=\overline{\mathbf{x}}^{1}$. This local optimization process is repeated, obtaining a sequence $\left\{\mathbf{x}^{k}\right\}$ of optimal points, until a convergence criteria is verified. We have experimentally verified in numerous tests, involving continuous functions to define the surface $\Sigma$, that this algorithm converges [5].

In order to prevent a loss of the details of the original geometry when we are smoothing the mesh, our algorithm evaluates the difference of heights $([\Delta z])$ between the centroid of the triangles of $M(p)$ and the reference surface, every time a new position $\mathbf{x}^{k}$ is calculated. If this distance exceeds a threshold, $\Delta(p)$, the movement of the node is aborted and the previous position is stored. This threshold $\Delta(p)$ is established attending to the size of the elements of $M(p)$. That is, the algorithm evaluates the average distance between the free node and the nodes connected to it, and takes $\Delta(p)$ as percentage of this distance. Other possibility is to fix $\Delta(p)$ as a constant for all local meshes. In the particular case in which we have an explicit representation of the surface by a function $f(x, y), \Delta(p)$ can be established as a percentage of the maximum difference of heights between the original surface and the initial mesh.

\section{Applications}

We present two examples that show the behavior of the projecting/smoothing technique. We have applied this technique 
to Igea (see Figure 7), http://www.cyberware.com/, and to a humerus, http://www.ulb.ac.be/project/vakhum/ (see Figure 10). The surface triangulation of Igea contains 67170 triangles and 33587 nodes and the humerus has 70000 and 35002 nodes.

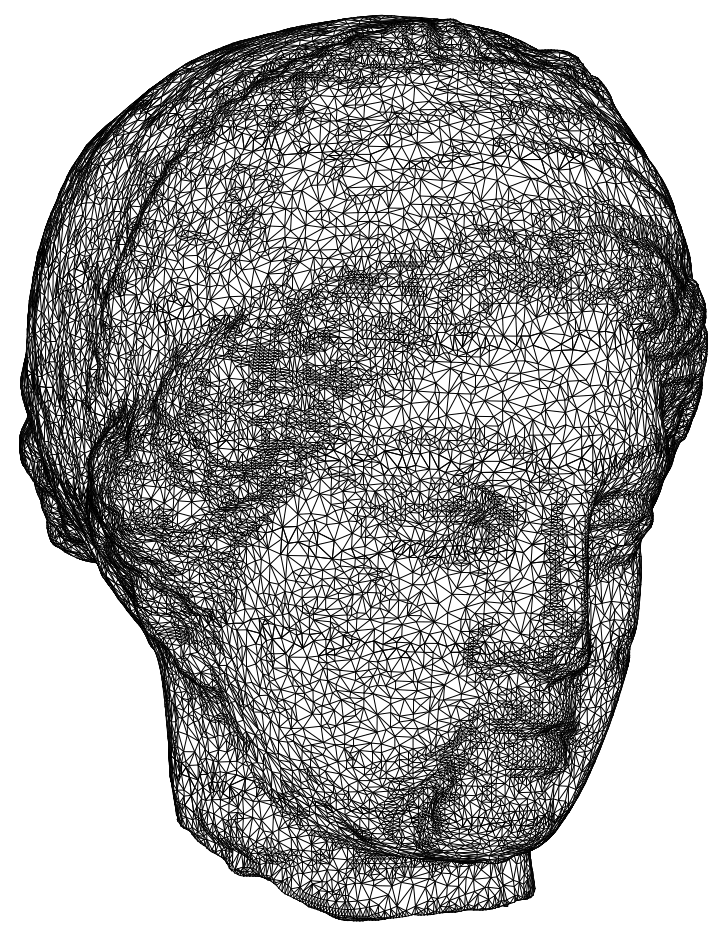

Fig. 7 Original mesh of Igea (http://www.cyberware.com/)

Our goal is to obtain a new triangulation (maintaining the initial topology) after applying the projecting/smoothing procedure to reach the alignment of the new mesh with the contours. As a first example we have chosen a mask and a star drawn on Igea's face. The second application is a plate, similar to the one used in traumatology, placed close to the humerus head. The contour of these objects is defined by splines which interpolate a few points placed in the parametric plane.

In Figure 8 we show the polylines that connect the interpolating points of the mask and the star. In order to keep the sharp angles, we have defined the star by using 10 splines linking the 10 interpolating points. Moreover, these points are enforced to be present in the final triangulation following the procedure pointed at the end of section 4.3. We propose the following strategy to get our objective.

Initially, we apply the smoothing technique [5] to the whole triangulation. In this case, the projection plane is chosen in terms of the local mesh to be optimized. The resulting mesh, after 4 iterations of our optimization procedure, is shown in Figure 9. The value of the average mesh quality (measured with the algebraic quality metric based on the condition number proposed in [9]) increases from 0.794 to 0.913. A more significant data is that average quality of the worst 100 triangles increases from 0.379 to 0.575 . We have fixed $\Delta(p)$ as $10 \%$ of average distance between the free node and the nodes connected to it. More details about this application can be seen in [18].

In order to reduce the computational cost of the alignment step, the projecting/smoothing process is carried out on the surface patches associated to the mask and to the star. For this purpose we select the set of triangles whose centroids are included in the reference windows of the mask and of the star. Both windows are marked with dashing lines in Figure 8. Then, we apply the projecting/smoothing procedure to these two sets separately. Note that, in the last process, the boundary of each patch triangulation is fixed, so we obtain an appropriate connection between the modified local meshes and the rest of the surface triangulation. These ideas could be used for a parallel implementation of the simultaneous aligning and smoothing local technique.

In Figure 10 it is presented a general view of the surface triangulation of Igea after applying 12 iterations of the aligning and smoothing procedure. The approximation of the contours by edges of the resulting triangulation is marked. After the application of our algorithm the values of minimum and average qualities become 0.100 (same value than in previous meshes) and 0.911 , respectively. The average

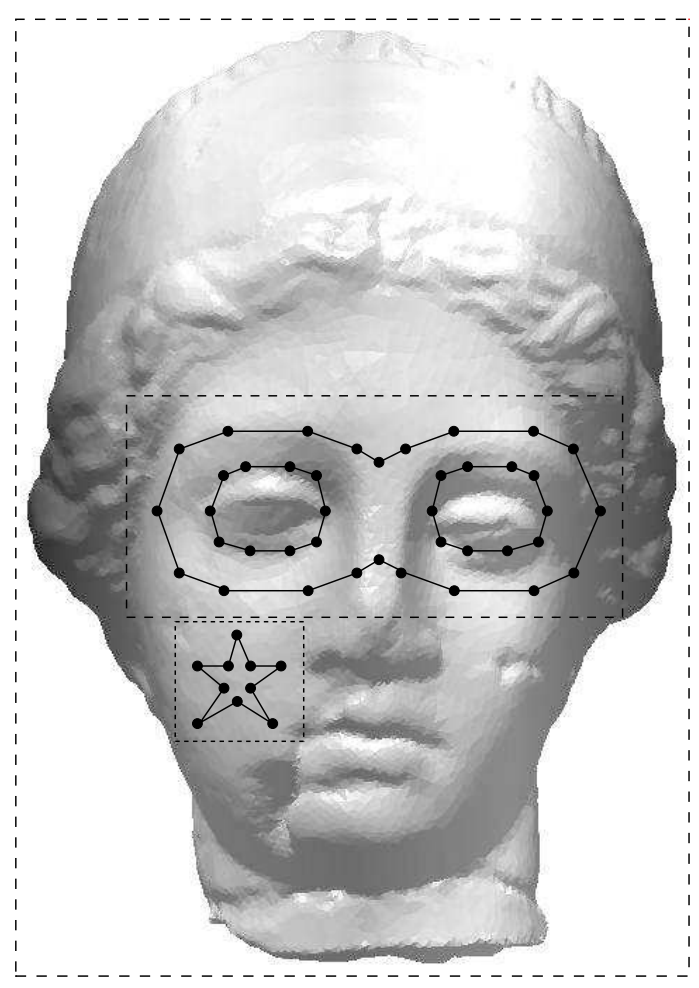

Fig. 8 Point input data for the definition of the curves, approximation of the splines as polylines and reference windows 


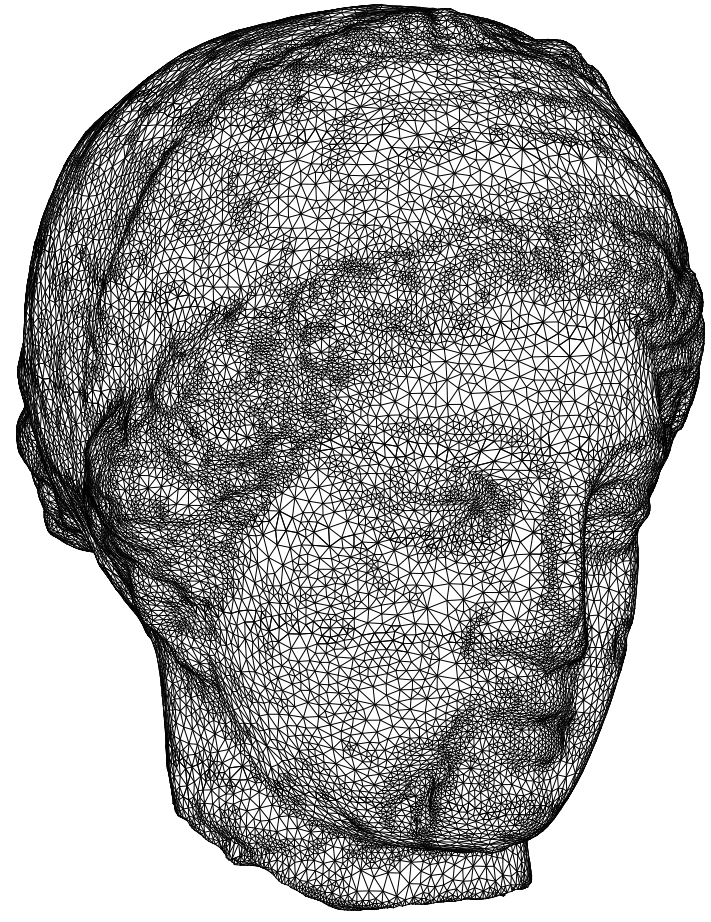

Fig. 9 Optimized mesh of Igea after 4 iterations of our smoothing procedure

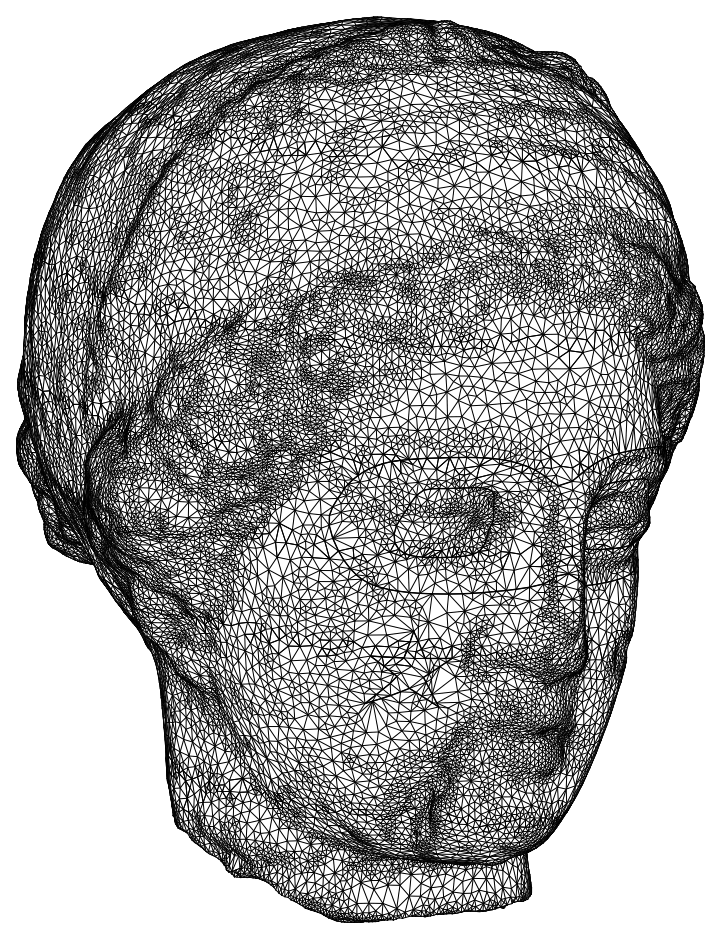

Fig. 10 Aligned and optimized mesh of Igea after 12 iterations of the local projecting/smoothing procedure

quality of the worst 100 triangles is 0.519 . Therefore, the mesh qualities are similar before and after the application of the projecting/smoothing technique.
Two details of the initial and final meshes (Figures 9 and 10) are shown in Figures 11(a) and (b), respectively. We represent the same marked edges before and after the local projecting/smoothing process. We note that it is very difficult to determine a priori which are the best edges for a suitable approximation of the contours of the mask and the star. However, the algorithm finds appropriate nodes (and consequently edges) automatically.

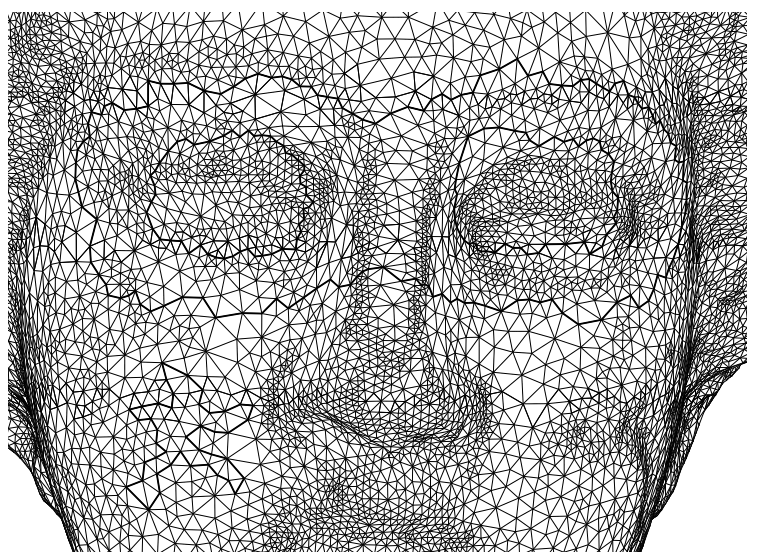

(a)

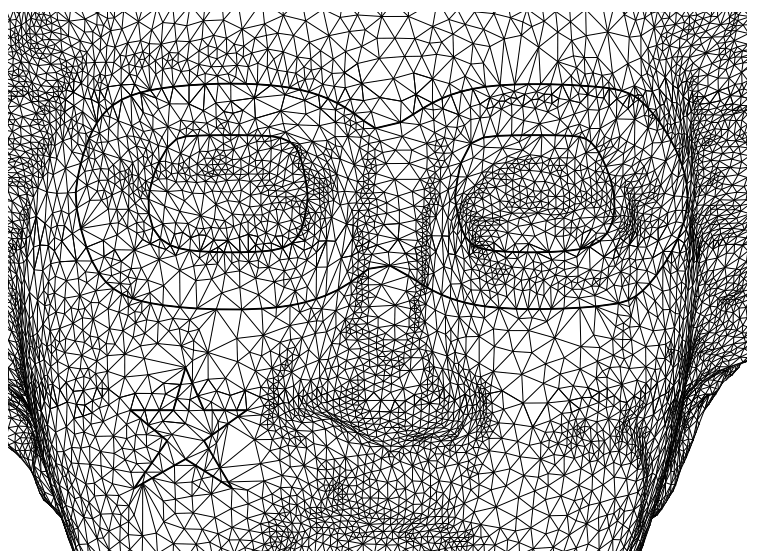

(b)

Fig. 11 Detail of the initial mesh of Igea with marked edges before projection (a). The same edges are remarked after the algorithm has projected them onto the contours of the mask and the star (b)

In Figure 12 (a) is shown the optimized mesh of the humerus after 5 iterations of the smoothing procedure applied to the whole triangulation. The mesh aligned to the plate, after 15 iterations of the projecting/smoothing procedure, is shown in Figure 12 (b). Actually, only 6 iterations of the last procedure have been necessary to get a continuous curve that interpolates the plate. The remaining iterations were done in order to smooth the mesh around the curve. The average quality of the worst 100 triangles before the application of the projecting/smoothing is 0.48 and, after, is 


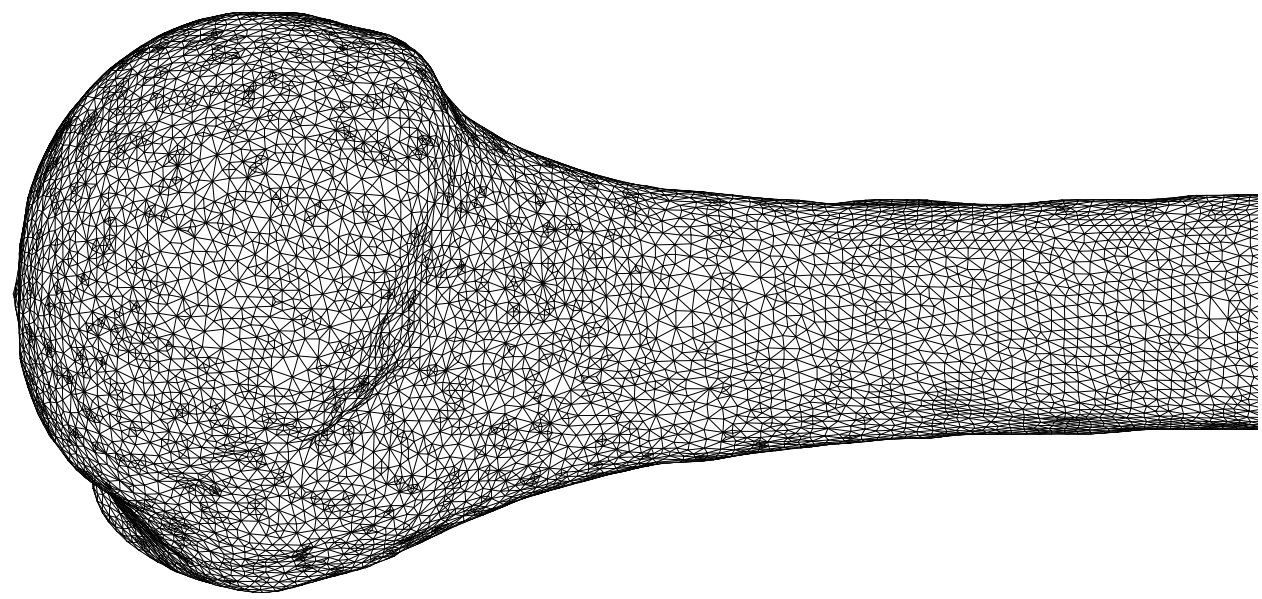

(a)

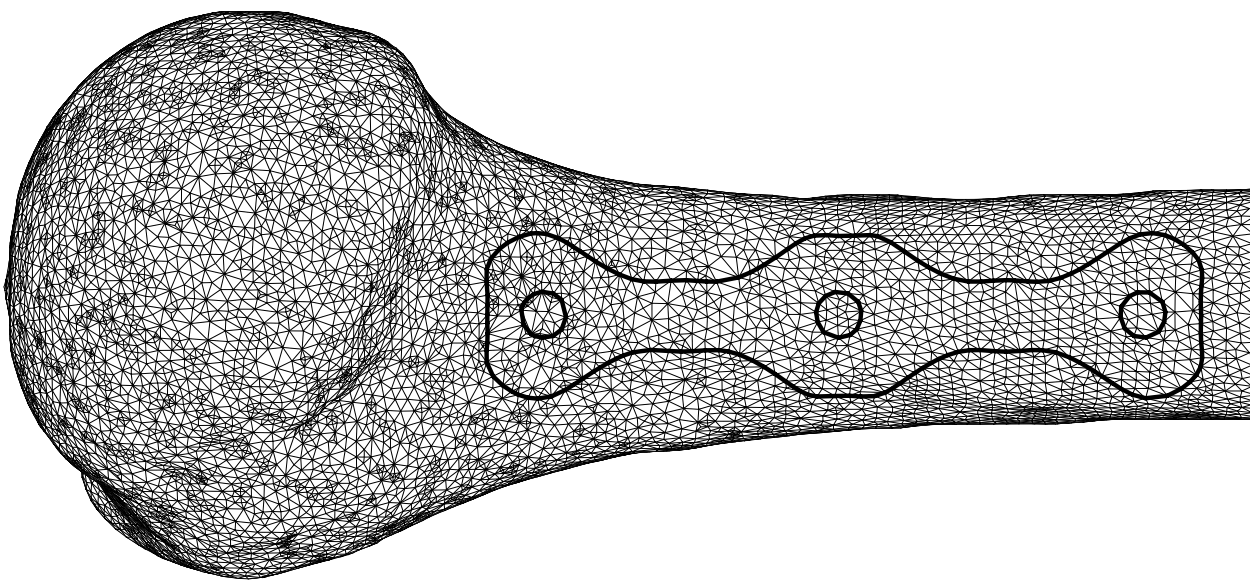

(b)

Fig. 12 Optimized mesh of the humerus after 5 iterations of the smoothing procedure (a). The aligned mesh after 15 iterations of the projecting/smoothing procedure (b)

0.47 , so this procedure has not a significant negative effect on the mesh quality.

\section{Concluding Remarks and Future Research}

In this paper we have introduced the projecting/smoothing technique which is able to align a surface triangulation with arbitrary curves without producing, in general, a significant decrease in the minimum quality of the mesh. Indeed, the average quality is increased in many cases as the remainder part of the mesh undergoes a smoothing process.

In present work the curves have been defined by splines whose interpolating points are fixed on a plane. Applications of this technique can be done in a straightforward manner, for example, in environmental modeling [17, 19] for aligning topographic surface meshes to significant contours, as coastlines, river banks, etc. In addition, this particular curve definition can be applied on different patches of a more general surface. Our method for aligning and smoothing of surface triangulations could be generalized by using a global parametric space (in similar terms as it is proposed in $[8,21]$ ) which makes the projection on a plane unnecessary. General parametric curves embedded on the surface will be considered. Another more ambitious generalization lies in extending the present method to align a tetrahedral mesh with interior surfaces. This is an open problem. It is clear that the mesh alignment problem is not always possible to solve. Generally, the existence of an admissible solution can not be assured because it depends on size, quality and topology of the initial mesh and regularity of the embedded curves.

Acknowledgements This work has been supported by the Secretaría de Estado de Universidades e Investigación of the Ministerio de Educación y Ciencia of the Spanish Government and FEDER, grant contract CGL2008-06003-C03-01. 


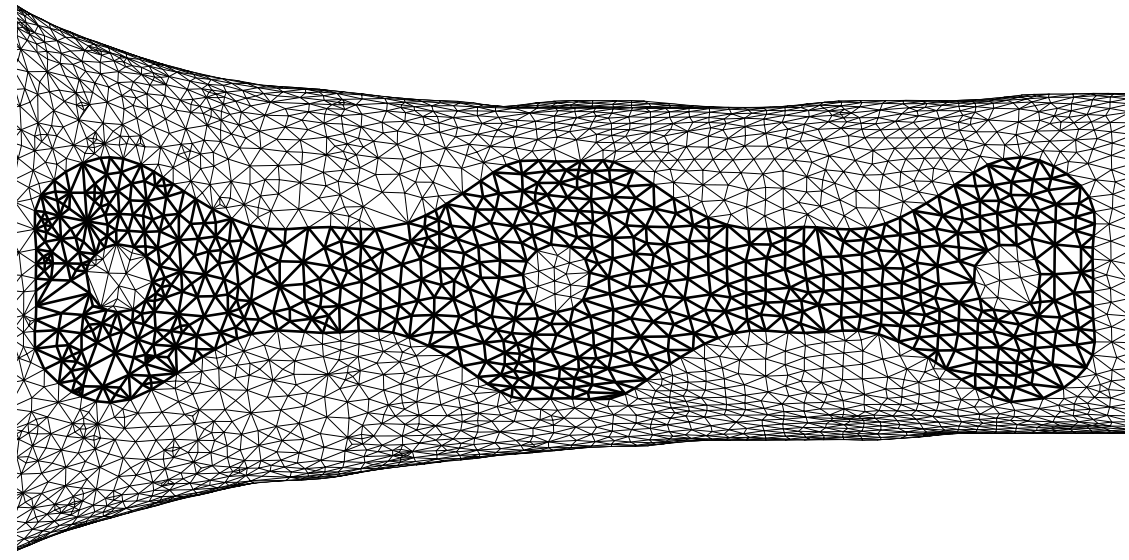

(a)

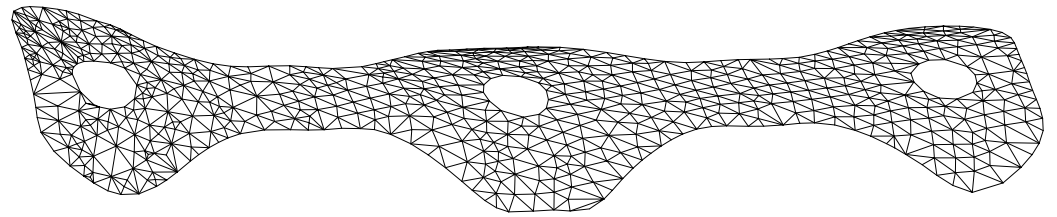

(b)

Fig. 13 Detail of the plate located on the humerus after projecting/smoothing (a). Another perspective of the isolated plate (b)

\section{References}

1. Bartels HR, Beatty JC, Barsky BA (1987) An introduction to Splines for use in computer graphics \& geometric modeling. Morgan Kaufmann, Los Altos, CA

2. Bazaraa MS, Sherali HD, Shetty CM (1993) Nonlinear programing: theory and algorithms. John Wiley and Sons Inc, New York

3. Bonneau GP, Hahmann S (2003) Smooth polylines on polygon meshes. In: Brunnett G, Hamann B, Mueller H (eds) Geometric modeling for scientific visualization 69-84. Springer, Berlin

4. Cascón JM, Montenegro R, Escobar JM, Rodríguez E, Montero G (2007) A new meccano technique for adaptive 3-D triangulations. In: Proceedings of the 16th Int Meshing Roundtable. Seattle, 103-120 October 2007.

5. Escobar JM, Montero G, Montenegro R, Rodríguez E (2006) An algebraic method for smoothing surface triangulations on a local parametric space. Int J Num Meth Eng 66:740-760

6. Escobar JM, Rodríguez E, Montenegro R, Montero G, GonzálezYuste JM (2003) Simultaneous untangling and smoothing of tetrahedral meshes. Comput Meth Appl Mech Eng 192:2775-2787

7. Escobar JM, Montenegro R, Rodríguez E, Montero G (2008) Simultaneous aligning and smoothing of surface triangulations. In: Proceedings of the 17th Int Meshing Roundtable. Pittsburgh, 333350, October 2008.

8. Floater MS (1997) Parametrization and smooth approximation of surface triangulations. Comput Aid Geom Design 14:231-250

9. Freitag LA, Knupp PM (2002) Tetrahedral mesh improvement via optimization of the element condition number. Int J Num Meth Eng 53:1377-1391

10. Frey PJ, Borouchaki H (1998) Geometric surface mesh optimization. Comput Visual Sci 1:113-121

11. Garimella RV, Shaskov MJ, Knupp PM (2004) Triangular and quadrilateral surface mesh quality optimization using local parametrization. Comput Meth Appl Mech Eng 193:913-928

12. González-Yuste JM, Montenegro R, Escobar JM, Montero G, Rodríguez E (2004) Local refinement of 3-D triangulations using object-oriented methods. Adv Eng Soft 35:693-702
13. Hyman JM, Li S, Knupp PM, Shashkov M (2000) An algorithm to align a quadrilateral grid with internal boundaries. J Comput Phys 163:133-149

14. Knupp PM (2000) Achieving finite element mesh quality via optimization of the Jacobian matrix norm and associated quantities. Part I - A framework for surface mesh optimization. Int J Num Meth Eng 48:401-420

15. Knupp PM (2000) Achieving finite element mesh quality via optimization of the Jacobian matrix norm and associated quantities. Part II - A framework for volume mesh optimization and the condition number of the Jacobian matrix. Int J Num Meth Eng 48:1165-1185

16. Knupp PM (2001) Algebraic mesh quality metrics. SIAM J Sci Comput 23:193-218

17. Montenegro R, Montero G, Escobar JM, Rodríguez E, GonzálezYuste JM (2002) Tetrahedral mesh generation for environmental problems over complex terrains. Lecture Notes in Computer Science 2329:335-344

18. Montenegro R, Escobar JM, Montero G, Rodríguez E (2005) Quality improvement of surface triangulations. 14th Int Meshing Roundtable 469-484. San Diego, California, USA.

19. Montero G, Rodríguez E, Montenegro R, Escobar JM, GonzálezYuste JM (2005) Genetic algorithms for an improved parameter estimation with local refinement of tetrahedral meshes in a wind model. Adv Eng Soft 36:3-10

20. Pav SE, Walkington NJ (2005) Delaunay refinement by corner looping. In: Proceedings of 14th Int Meshing Roundtable. San Diego 165-181, September 2005.

21. Sheffer A, De Sturler E (2002) Smoothing an overlay grid to minimize linear distortion in texture mapping. ACM Trans Graph 21:874-890

22. Stein K, Tezduyar TE, Benney R (2004) Automatic mesh update with the solid-extension mesh moving technique. Comput Meth Appl Mech Eng 193:2019-2032 